\title{
Study protocol for a randomized controlled trial
} of telephone-delivered cognitive behavior therapy compared with befriending for treating depression and anxiety in older adults with COPD

This article was published in the following Dove Press journal:

International Journal of COPD

12 February 2016

Number of times this article has been viewed

\section{Colleen Doyle,' David \\ Dunt, ${ }^{2}$ David Ames, ${ }^{3}$ Marcia \\ Fearn, ${ }^{3}$ Emily (Chuanmei) \\ You,' Sunil Bhar ${ }^{4}$ \\ 'Australian Catholic University, Melbourne, VIC, Australia; ${ }^{2}$ Centre for Health Policy, The University of Melbourne, Melbourne, VIC, Australia; ${ }^{3}$ National Ageing Research Institute, Melbourne, VIC, Australia; ${ }^{4}$ Department of Psychological Sciences, Swinburne University of Technology, Hawthorn, VIC, Australia}

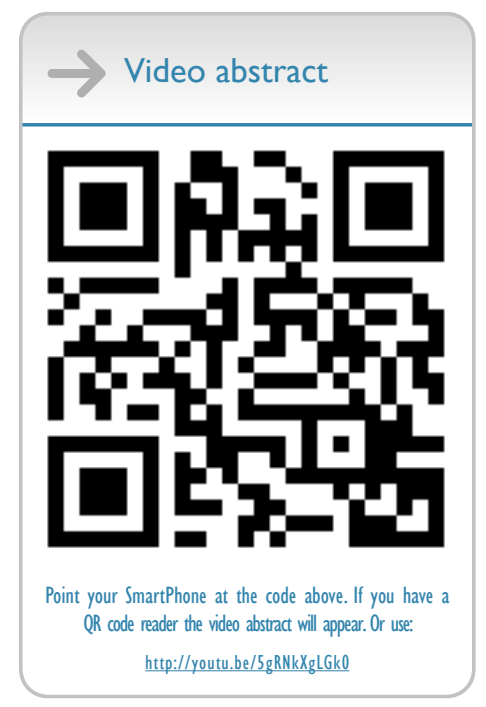

Correspondence: Colleen Doyle Villa Maria Catholic Homes, 6 Studley Park Rd, Kew, VIC 3I0I, Australia

Tel +6I 409739136

Fax +6I 393874030

Email colleen.doyle@acu.edu.au

\begin{abstract}
Background: COPD is an umbrella term to describe chronic lung diseases that cause limitations in lung airflow, including emphysema and chronic bronchitis. The prevalence of depression and anxiety in people with COPD is high, although these comorbidities are often undiagnosed, untreated, or undertreated. There is a need to identify efficacious treatments for depression and anxiety in people with COPD. Cognitive behavior therapy (CBT) for the treatment of anxiety and depression has a strong evidence base. There has been some success delivering this treatment over the telephone in limited studies. The aim of this study is to evaluate the efficacy of both telephone-administered CBT and befriending on outcomes for patients with diagnosed COPD who have at least mild levels of depression and/or anxiety.
\end{abstract}

Methods: The protocol described in this paper is of a pragmatic randomized controlled trial comparing eight sessions of telephone CBT to an active social control, referred to as befriending. Primary outcome measures will include depression and anxiety symptoms, and secondary outcome measures will include quality of life, self-efficacy, and COPD symptom severity. Participants' satisfaction with the intervention and therapeutic alliance will also be assessed. Measures will be taken pre- and postdelivery of the intervention and again at 8 weeks following the intervention. Conclusion: People with COPD often have limitations to their mobility because of their breathlessness. They are often already attending many medical appointments and could be reluctant to attend for face-to-face psychological treatment. The results of this study should identify the relative efficacy of CBT delivered over the telephone to this population, which, if successful, may be a cost-effective and more palatable alternative to face-to-face treatment of depression or anxiety for this population.

Keywords: chronic obstructive pulmonary disease (COPD), depression, anxiety, telephone, cognitive behavior therapy (CBT), befriending

\section{Background}

COPD is a long-term lung disease characterized by airflow obstruction and shortness of breath, which progressively limit activities of daily living. In addition to shortness of breath, other symptoms of COPD include wheezing, coughing, mucus, fatigue, cyanosis, and increased susceptibility to chest infections. An estimated 2 million Australians are affected by COPD, ${ }^{1}$ with 1.2 million having moderate-to-severe COPD.

COPD has multiple comorbidities, and two of the most common and least-treated comorbidities are depression and anxiety. ${ }^{2}$ There has been no consensus on the prevalence of depression and anxiety in people with COPD, due in part to the variation in 
measurement tools and the severity of illness in different studies. However, it has been estimated that one in four people with COPD experience clinically significant depressive symptoms, and up to $40 \%$ of people with COPD suffer from clinical anxiety. ${ }^{3}$

The exact cause of depression and anxiety in people with COPD is unknown; however, some of the variables that have been implicated include physical disability, long-term oxygen therapy, low body mass index, severe dyspnea, percentage of predicted forced expiratory volume in 1 second $\left(\mathrm{FEV}_{1}\right)$, poor quality of life, presence of comorbidities, living alone, female sex, current smoking, and low social class status. ${ }^{2}$

Although depression and anxiety are common in patients with COPD, they are often undetected, undertreated, or even untreated. ${ }^{2}$ This can lead to increased mortality risk, reduced compliance with management plans, impaired healthrelated quality of life, slower recovery from exacerbations, and increased health-care utilization and costs. ${ }^{2-4}$

Regarding the treatment options of depression and anxiety in patients with COPD, researchers have suggested promoting nonpharmacological interventions as the firstline therapy. Of those, cognitive behavior therapy (CBT) has been offered by trained CBT therapists or other health professionals, such as community nurses. ${ }^{5-7}$ CBT is a type of psychological therapy or counseling technique that helps a person change unhelpful or unhealthy thinking, their thoughts or cognitions, and also their actions or behaviors. Previous systematic reviews have reported that there is some support for using this therapy to improve the condition of people with COPD, reducing their anxiety and depression levels; 5,8 however, there is debate about the evidence that is largely derived from either small, randomized controlled trials or uncontrolled or nonrandomized studies. ${ }^{5}$

People with COPD can often be disabled and have physical limitations, which may impact on their ability to travel to various medical appointments. Physical mobility restrictions and issues with transport have been reported as factors preventing people from participating in intervention trials. ${ }^{9}$ It has been reported that approximately $75 \%$ of depressed primary care patients report barriers that make it difficult for them to attend regular psychotherapy sessions. ${ }^{10}$ These barriers include lack of accessible services, transportation services, and cost.

Telephone-administered CBT allows people to receive counseling therapy in their own homes. It has lower attrition rates compared to face-to-face $\mathrm{CBT}^{10}$ and has been found to be as effective in reducing depression and anxiety as faceto-face CBT. ${ }^{10,11}$ Telephone-administered CBT may provide those people with COPD who have physical limitations that prevent them from attending medical appointments at a hospital or medical center, with the opportunity to receive counseling therapy.

\section{Study aims and objectives}

\section{Aim}

The aim of this trial is to evaluate the efficacy of telephoneadministered CBT compared with an active social controlbefriending - on outcomes for patients with diagnosed COPD who have at least mild levels of depression and/or anxiety.

\section{Objectives}

The study objectives are to:

1) Investigate changes to levels of depression and anxiety in participants before and after the telephone intervention and at follow-up and compare these between the groups receiving the $\mathrm{CBT}$ intervention with those receiving befriending;

2) Investigate changes to secondary outcome measures such as self-efficacy, quality of life, and COPD symptom severity; and

3) Evaluate therapeutic alliance and participants' level of satisfaction with the intervention they receive.

\section{Methods}

\section{Study design}

This study is a pragmatic randomized controlled trial. ${ }^{12}$ It includes a telephone screen (conducted by the researchers using standardized screening scales) for identifying eligible participants, baseline assessment for assessing enrolled participants' health and well-being, randomized allocation of the participants to the intervention (CBT) or control (befriending) group, initial session to build rapport, 8 weekly telephone interventions of either CBT or befriending, a postintervention follow-up assessment, and another follow-up assessment 8 weeks after the intervention (Figure 1). The study is blind to the researchers undertaking the baseline and follow-up assessments, and the data analysis. Owing to the nature of the intervention and control conditions, the therapists and volunteers delivering the interventions and the participants are not able to be blind to the conditions.

\section{Ethical considerations}

Ethical approvals for the trial have been obtained from Melbourne Health, Alfred Health, Austin Health, Barwon Health, Eastern Health, and Bendigo Health. 


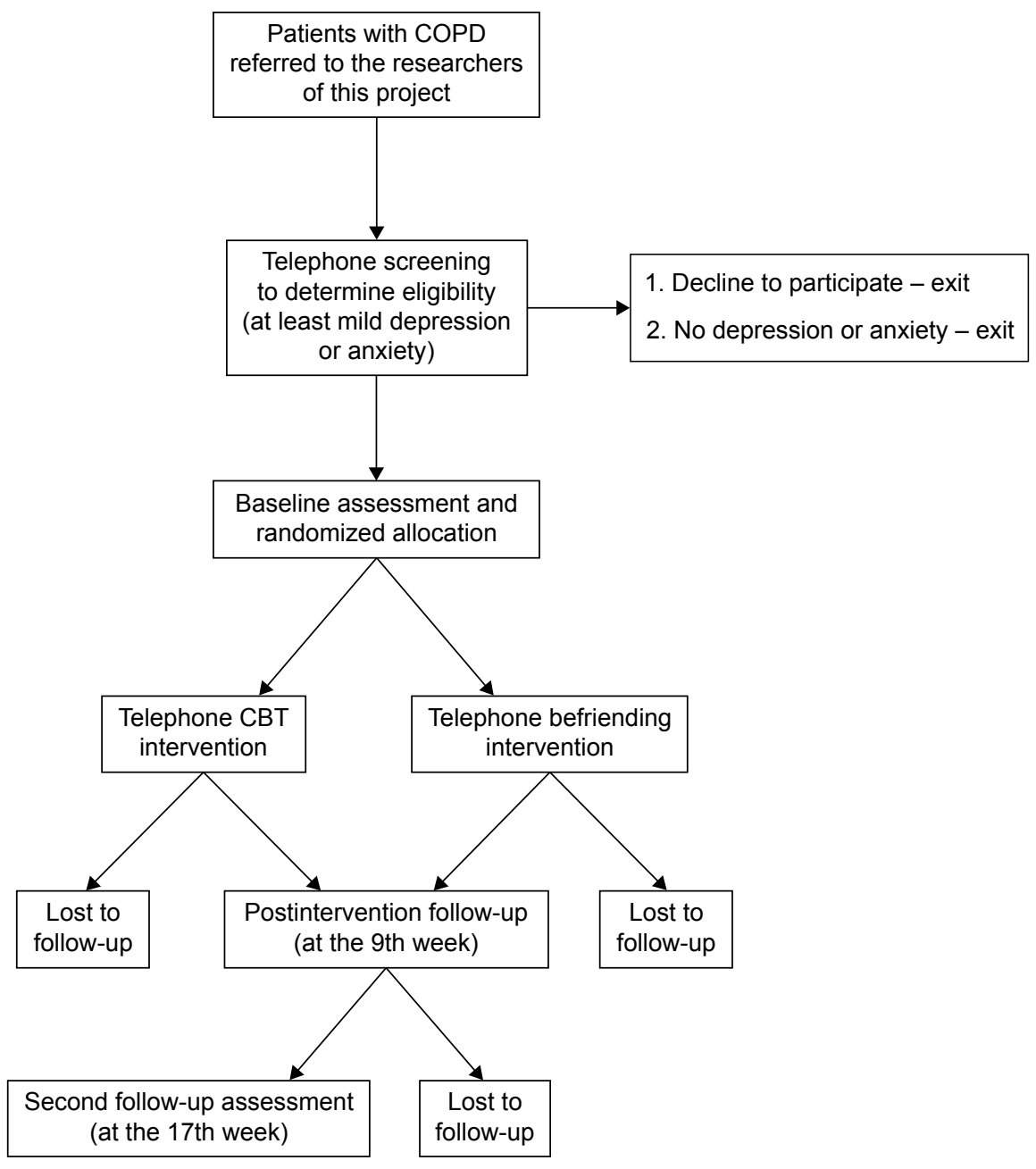

Figure I Study design.

Abbreviation: $\mathrm{CBT}$, cognitive behavioral therapy.

The trial is registered with the ANZ trial register (ACTRN12612000254897).

\section{Study procedures}

\section{Power and sample size}

Using the effect sizes published in a systematic review of the impact of CBT on depression and anxiety that were measured by different instruments such as Symptom Checklist-90Revised (SCR-90-R), Geriatric Depression Scale (GDS), Beck Depression Inventory (BDI), and Beck Anxiety Inventory (BAI) in the included studies, ${ }^{8}$ it is expected that there will be standardized mean difference effect size between CBT and usual care of $0.5-0.6$ on this outcome (calculated using the METAN procedure from Stata 11 using published effect sizes for depression and anxiety). It is reasonable to assume that telephone $\mathrm{CBT}$ will provide a similar effect size to that found in studies of face-to-face CBT. For a study with $80 \%$ power and alpha error probability of 0.05 , we will require at least 65 patients per group. Assuming loss to follow-up of $5 \%-10 \%$, we will aim to have 70 patients in each group. On a conservative estimate based on prevalence figures published in the literature and our current study, it is expected that $40 \%$ of patients referred to the outpatient clinics will have at least mild depression and/or anxiety; so 350 patients may need to be screened to provide the sample size of 140 altogether. In a previous study, we recruited 103 people with COPD attending 22 pulmonary rehabilitation (PR) clinics into the study over a 12 -month period: ${ }^{13}$ so we estimate that 30 months should be sufficient time to recruit the 140 clients required for this study.

\section{Participant inclusion criteria}

Eligible participants will be patients with a diagnosis of COPD together with the following characteristics: 1) living in the community; 2) aged 45 years or older; 3) having a telephone and being able to hear over the phone; 
4) understanding and speaking English; 5) having been on a stable dose for at least 3 months if on psychotropic medication; and 6) scoring 8 or more on the Hospital Anxiety and Depression Scale (HADS) or 10 or more on the Patient Health Questionnaire-9 (PHQ-9). Because of its brevity, HADS will be used during the telephone screening to determine eligibility of the patients.

Notably, where possible, diagnosis of COPD will be confirmed by participants' general physicians. For participants who are self-referred from the Newsletters or Website, their diagnosis will be assumed from self-report, COPD Assessment Test (CAT) result, and lung function tests.

\section{Recruitment and baseline assessment}

Participants will be recruited from the outpatient clinics of respiratory departments of tertiary hospitals in Victoria, from Pulmonary Rehabilitation Programs across the Melbourne metropolitan area, and from advertisements on patient support group websites. In the first two recruitment sources, patients with a diagnosis of COPD will be identified and then referred to the researchers of this study by a member of the team providing respiratory health care. The researchers will then assess each patient's eligibility for this study through conducting a telephone screening.

For all participants recruited, the researchers will undertake the formal consent process during the introduction session. Information and consent forms will be posted to eligible participants, then written formal consent will be obtained at the participant's home or a public place where the introduction session is conducted. If the introduction session is to be conducted over the phone, participants will be instructed to mail their consent forms back to the researchers, and the intervention will not commence until consent forms are received. The researchers will fully explain the nature of the study to the participants, including their right to withdraw consent at any time. Once the participant's informed consent is obtained, the baseline assessment will be obtained by using the assessment tools outlined in Table 1.

\section{Randomization}

Following the baseline assessment, participants will be randomly allocated to the CBT or befriending condition. Randomization will be managed by a trial statistician who is not directly involved in the project. A computer-generated random sequence available only to the trial statistician will be used to produce randomized allocation slips. The latter will use a randomized permuted block design with block size of 4,6 , or 8 in order to maintain approximately balanced randomization throughout the trial. Participants will then be contacted by their telephone support person, and an introductory session will be arranged either at the participant's home, in a public space such as a coffee shop (if the participant refuses a home visit), or over the phone (if the participant lives in remote area), in order to reinforce the procedure, introduce the telephone support person, explain the project, and establish rapport.

\section{Telephone CBT and befriending interventions}

The 8 weekly telephone interventions/sessions of the CBT and befriending will then commence. Up to ten therapists (providing CBT sessions) and up to ten volunteers (providing befriending sessions) will participate in this study considering the participant flow and availability of therapists and volunteers. Variability between providers will be controlled through training and quality monitoring by the chief investigators (SB and CD) of this study. Details about the telephone interventions are described in the following sections.

\section{Cognitive behavior therapy}

The treatment program will include behavioral strategies such as behavioral activation, activity scheduling, relaxation training, exposure hierarchies, and social skills training, as well as cognitive strategies, such as cognitive restructuring,

Table I Outcome measures used at different assessment points in the study

\begin{tabular}{|c|c|c|c|c|c|}
\hline Domains & Primary or secondary measure & Instrument & $\mathbf{T I}^{\mathbf{a}}$ & $\mathbf{T}^{\mathrm{b}}$ & $\mathbf{T 3}^{\mathrm{c}}$ \\
\hline \multirow[t]{4}{*}{ Health and well-being } & Depression (primary) & Patient Health Questionnaire-9 & $\checkmark$ & $\checkmark$ & $\checkmark$ \\
\hline & Anxiety (primary) & Beck Anxiety Inventory & $\checkmark$ & $\checkmark$ & $\checkmark$ \\
\hline & Health-related quality of life (secondary) & The COPD Assessment Test & $\checkmark$ & $\checkmark$ & $\checkmark$ \\
\hline & Self-efficacy (secondary) & General Self-Efficacy Scale & $\checkmark$ & $\checkmark$ & $\checkmark$ \\
\hline \multirow[t]{2}{*}{ Intervention response } & Participant satisfaction & Client Satisfaction Questionnaire & & $\checkmark$ & \\
\hline & Therapeutic alliance & Working Alliance Inventory-Short & & $\checkmark$ & \\
\hline \multirow[t]{2}{*}{ Demographic and health-related Information } & Stable demographic factors & Interview & $\checkmark$ & & \\
\hline & Variable demographic factors and health factors & Interview & $\checkmark$ & $\checkmark$ & $\checkmark$ \\
\hline
\end{tabular}

Note: ${ }^{a}$ Baseline assessment, ${ }^{b}$ postintervention assessment, cfollow-up assessment 8 weeks after the CBT/befriending intervention.

Abbreviation: $\mathrm{CBT}$, cognitive behavior therapy. 
structured problem solving, and behavioral experiments. Interventions will be delivered from a treatment protocol but be flexibly tailored to the conceptualization of the individual's presenting problem. Following an initial session getting to know one another, eight sessions of telephoneadministered CBT are offered. Specific topics of the eight therapy sessions will be as follows: session 1 "depression and activity tracking"; session 2 "activity scheduling"; session 3 "relaxation skills"; session 4 "cognitive restructuring"; session 5 "problem solving"; session 6 "sleep management"; session 7 "review and practice coping skills"; and session 8 "maintaining gains and goodbye". A manual (available from authors) that introduces these sessions and the procedures of conducting the sessions has been developed based on previous academic clinical trials that demonstrate efficacy of the procedures for older adults with anxiety ${ }^{14-16}$ and younger patients with depression. ${ }^{17,18}$

The telephone calls will occur weekly, at a time convenient to both the therapist and participant and will be of approximately 30 minutes in duration. The therapist delivering the intervention will be a registered or provisionally registered psychologist experienced in the delivery of telephone CBT and with knowledge of COPD education. Integrity of interventions and consistency of treatment across sites as indicated in the manual will be maintained with the use of a treatment protocol, therapist competency audits, training workshops for therapists, and ongoing supervision by a clinical psychologist. The CBT telephone calls will be audio recorded to assist with such monitoring.

\section{Active social control: befriending}

The control group will be provided with a course of befriending. Befriending will focus on neutral topics and will avoid discussion of symptoms or difficulties in client's lives. In previous studies, ${ }^{19}$ befriending has been used to control for the factors of time and therapeutic alliance that could arise as a result of CBT in a randomized controlled trial of CBT for treatment-resistant psychosis, but it has not been used for participants with COPD. Previous studies showed that befriending was effective in reducing symptoms in people with psychosis but less so than CBT at 1-year follow-up. Such studies suggested that spending time talking to and then developing a relationship with a person may be seen to be as beneficial in the short-term to the person as the treatment from the CBT. ${ }^{20} \mathrm{~A}$ key characteristic of befriending is that it can be administered by individuals without psychological or medical training; thus it is potentially cheaper to provide.

Befriending involves a series of conversations with a person similar to those that one would have with a social acquaintance, involving discussion of neutral, everyday topics and events in a friendly manner. There is no problem solving or exploration of emotions in befriending.

In this study, the befriending condition will consist of one introductory session, followed by eight scheduled weekly telephone calls. Each telephone call will last approximately 30 minutes and be audio recorded to enable monitoring. The befrienders (volunteers) will be provided with training and a manual on befriending (adapted from Bendall et al's work ${ }^{19}$ and available from authors). The manual includes guidelines around focusing on neutral topics, developing a therapeutic relationship, introducing and orienting a client to befriending, what to do if the client deviates from neutral topics, and how to manage client distress, and confidentiality and safety issues for both the befriender and the client.

\section{Follow-up assessments}

Following the telephone interventions, a postintervention assessment will occur for each participant. Besides the same assessment tools as those used in the baseline assessment, two additional assessment tools will be used to assess participants' response to the interventions (Table 1). In addition, 8 weeks after the interventions, the researchers will conduct a second follow-up assessment by using the measurement tools presented in Table 1.

\section{Outcomes and measures}

A range of outcome measures will be used at different assessment points. These measures will assess emotional well-being (depression, anxiety, and self-efficacy), physical well-being relating to living with COPD (health status), participant satisfaction with the intervention, and the therapeutic alliance. In addition, participants will be asked for basic demographic information and a self-rating of other health problems (Table 1).

\section{Health and well-being outcome measures Depression (primary outcome)}

The PHQ-9 will be used to assess the level of depression in participants. It is a 9-item depression scale based on the Diagnostic and Statistical Manual of Mental Disorders, fourth edition (DSM-IV) criteria for depression and is designed as both a diagnostic instrument and a severity measure. ${ }^{21}$ It has good internal consistency and test-retest reliability and criterion validity. ${ }^{21}$ It is one of the instruments recommended for use in primary care in the UK to assess depression severity. ${ }^{22}$ Scores can range from 0 to 27 , with higher scores reflecting more severe depression. The PHQ-9 
has also demonstrated sensitivity to change over time and in response to treatment. ${ }^{23,24}$

\section{Anxiety (primary outcome)}

The BAI will be used to assess the level of anxiety in participants. ${ }^{25}$ The BAI is a 21-item scale designed to measure the severity of anxiety in adults and adolescents. It has also been demonstrated to have sound psychometric properties when used in older adults. ${ }^{25}$

\section{Health status (secondary outcome)}

The COPD Assessment Test (CAT) is a validated instrument used to measure the impact of COPD. ${ }^{26}$ It is an eight-item self-report questionnaire that is simple and quick to complete and helps to assess the health status of people with COPD. It has been shown to be responsive to changes in health status resulting from exacerbations of the disease and treatment such as PR. ${ }^{27,28}$ Each item is scored from 0 to 5, representing the extent of the impact of that particular item, resulting in an overall score ranging from 0 to 40 . Higher scores reflect greater impact of the disease, and changes of 2 points or more suggests a clinically significant difference. ${ }^{28}$

\section{Self-efficacy (secondary outcome)}

The General Self-Efficacy Scale is a 10-item scale designed to assess perceived self-efficacy. It is a measure of coping and adaptation and will be used to identify any impact of the intervention on these variables. Total scores can range from 10 to 40, with higher scores reflecting greater perceived self-efficacy.

\section{Intervention response measures}

Participant satisfaction

The acceptability of the intervention and participant satisfaction will be assessed with the Client Satisfaction Questionnaire, ${ }^{29}$ which is an 8-item measure with good internal consistency widely used to assess patient satisfaction with services. Responses to each item are made on a fourpoint Likert scale, with higher scores representing a greater level of satisfaction.

\section{Therapeutic alliance}

Therapeutic alliance will be measured with the Working Alliance Inventory (WAI)-S. Therapeutic alliance is seen to be critical in the success of different forms of psychotherapy, with evidence demonstrating a relationship between therapeutic alliance and treatment outcome. ${ }^{30}$ Horwath and Greenberg $^{31}$ developed the original WAI with both therapist and client versions, and this 36-item instrument was later shortened to 12 items by Tracey and Kokotovic. ${ }^{32}$ The WAI-S is a self-report questionnaire that measures therapeutic alliance and its relationship to the success or failure of an intervention. Three key aspects of the therapeutic alliance are assessed: 1) agreement on the tasks of therapy; 2) agreement on the goals of therapy; and 3) development of an affective bond, each of which is represented by a subscale comprising four items each. Items are scored on a 7-point Likert-type scale ranging from 0 (never) to 7 (always), with higher scores reflecting a more positive rating of working alliance. Both total scale scores and subscale scores have been demonstrated to be reliable. ${ }^{33}$

\section{Demographics and health-related information}

A range of demographics and health-related information will be collected from participants in order to describe the characteristics of the participant sample. These will include: 1) age, sex, marital status, postcode, living arrangements, ethnicity, education level, occupation, and financial status; 2) self-rated health, severity of medical conditions (measured by the Charlson Index ${ }^{34}$ ), date of diagnosis of COPD, participation in PR program, and COPD severity (measured by average of three spirometer readings of $\mathrm{FEV}_{1}$ ).

\section{Data analysis}

\section{Statistical analyses}

Analysis will be on an intention-to-treat basis and reported in accordance with the revised CONSORT statement. ${ }^{35}$ The adequacy of the randomization procedures will be assessed by comparison of all baseline characteristics between the two study groups. The statistical test used to compare differences in mean depression and mean anxiety score between baseline and follow-up in the two groups will depend on the degree of skewness of the distribution of scores. If distributions are skewed, the nonparametric Mann-Whitney will be used, otherwise a simple two-sample $t$-test will be used. No adjustment will be made for multiple comparisons since the overall number of comparisons involving the outcome variable will be small.

\section{Discussion}

To our knowledge, this is the first randomized controlled trial that evaluates the efficacy of both telephone-administered CBT and befriending on outcomes for patients with COPD who also have at least mild levels of depression and/ or anxiety. There has been evidence showing that compared with face-to-face CBT, telephone CBT can achieve 
comparative clinical effects ${ }^{11}$ or some better results, such as improving patients' adherence to treatment. ${ }^{10,36}$ Therefore, in our study, we expect that the telephone CBT will reduce the levels of depression and anxiety and improve self-efficacy, quality of life, and COPD symptom severity in participants with COPD.

Telephone CBT is promising for patients with COPD and associated depression and anxiety because provision of faceto-face CBT (at home, the clinic, or other locations) might be constrained by time, space, costs (such as travel costs), and patients' abilities to access transport and care services. ${ }^{37}$ If the current study proves the positive effects of telephone CBT, this model of intervention has a great potential to serve as prototype for practice of care professionals.

Methodological strengths of this study are as follows: first, a trial statistician conducting the computerized randomization procedure ensures chance allocation of participants to the intervention or control group; second, the choice of the primary and secondary outcomes and measurement tools is based on the best evidence available, enabling us to comprehensively evaluate the efficacy of telephone-administered CBT; third, by assessing the medium-term (at the 17-week follow-up) outcomes, we can identify differences in the effects of telephone-administered CBT and befriending that might emerge in the weeks following the completion of the interventions.

One limitation of this study was that we were not able to blind the therapists, volunteers, or participants, which might affect the intervention effects. For example, some participants might feel discouraged to be allocated to the control group; therefore, they would be less responsive to the befriending intervention. Another limitation might be related to the low participant representativeness by using the current recruitment strategy. However, this is a recurring issue facing many randomized control trials. Finally, although in our study we examined medium-term (at the 17-week follow-up) intervention effects, it is unclear whether this is the optimum duration. Another longer-term follow-up is outside the funding of this study.

The findings of this study will add to the understanding of the role that telephone CBT may play in assisting patients with COPD who have significant levels of depression and anxiety, as well as suggest an appropriate model for trials of CBT in a wider range of population of this kind.

\section{Acknowledgments}

This study was funded by beyondblue and was undertaken with assistance from the National Ageing Research Institute
(NARI). Alex Gorelik assisted with statistical estimates. Arthur Hsueh, Chris Chiu, and Maree Daly assisted with design discussions.

\section{Author contributions}

All authors contributed toward data analysis, drafting and revising the paper and agree to be accountable for all aspects of the work.

\section{Disclosure}

The authors report no conflicts of interest in this work.

\section{References}

1. Access Economics. Economic Impact of COPD and Cost Effective Solutions. Milton, QLD: Australian Lung Foundation; 2008.

2. Maurer J, Rebbapragada V, Borson S, et al. Anxiety and depression in COPD: current understanding, unanswered questions, and research needs. Chest. 2008;134(4 Suppl):43S-56S.

3. Panagioti M, Scott C, Blakemore A, Coventry PA. Overview of the prevalence, impact, and management of depression and anxiety in chronic obstructive pulmonary disease. Int J Chron Obstruct Pulmon Dis. 2014;9:1289-1306.

4. Fritzsche A, Clamor A, von Leupoldt A. Effects of medical and psychological treatment of depression in patients with COPD - a review. Respir Med. 2011;105(10):1422-1433.

5. Coventry PA, Bower P, Keyworth C, et al. The effect of complex interventions on depression and anxiety in chronic obstructive pulmonary disease: systematic review and meta-analysis. PLoS One. 2013; 8(4):e60532.

6. Heslop K, Newton J, Baker C, Burns G, Carrick-Sen D, De Soyza A Effectiveness of cognitive behavioural therapy (CBT) interventions for anxiety in patients with chronic obstructive pulmonary disease (COPD) undertaken by respiratory nurses: the COPD CBT CARE study: (ISRCTN55206395). BMC Pulm Med. 2013;13:62.

7. Barker D, Davies C, Dixon B, Hodgson A, Reay S, Barclay N. Training community matrons in basic cognitive behavioural therapy-based techniques for patients with COPD. Br J Community Nurs. 2014;19(6): 274-283.

8. Coventry PA, Gellatly JL. Improving outcomes for COPD patients with mild-to-moderate anxiety and depression: a systematic review of cognitive behavioural therapy. Br J Health Psychol. 2008;13(Pt 3): 381-400.

9. Elzen H, Slaets JP, Snijders TA, Steverink N. Do older patients who refuse to participate in a self-management intervention in the Netherlands differ from older patients who agree to participate? Aging Clin Exp Res. 2008;20(3):266-271.

10. Mohr DC, Ho J, Duffecy J, et al. Effect of telephone-administered vs face-to-face cognitive behavioral therapy on adherence to therapy and depression outcomes among primary care patients. JAMA. 2012; 307(21):2278-2286.

11. Hammond GC, Croudace TJ, Radhakrishnan M, et al. Comparative effectiveness of cognitive therapies delivered face-to-face or over the telephone: an observational study using propensity methods. PLoS One. 2012;7(9):1-15.

12. Hotopf M. The pragmatic randomised controlled trial. Adv Psychiatr Treat. 2002;8(5):326-333.

13. Doyle C, Dunt D, Ames D, Selvarajah S. Managing mood disorders in patients attending pulmonary rehabilitation clinics. Int J Chron Obstruct Pulmon Dis. 2013;8:15-20.

14. Stanley MA, Diefenbach GJ, Hopko DR. Cognitive behavioral treatment for older adults with generalized anxiety disorder. A therapist manual for primary care settings. Behav Modif. 2004;28(1):73-117. 
15. Stanley MA, Novy DM. Cognitive-behavior therapy for generalized anxiety in late life: an evaluative overview. J Anxiety Disord. 2000; 14(2):191-207.

16. Stanley MA, Beck JG, Glassco JD. Treatment of generalized anxiety in older adults: a preliminary comparison of cognitive-behavioral and supportive approaches. Behav Ther. 1996;27(4):565-581.

17. Jacobson NS, Dobson KS, Truax PA, et al. A component analysis of cognitive-behavioral treatment for depression. J Consult Clin Psychol. 1996;64(2):295-304.

18. Gortner ET, Gollan JK, Dobson KS, Jacobson NS. Cognitive-behavioral treatment for depression: relapse prevention. J Consult Clin Psychol. 1998;66(2):377-384.

19. Bendall S, Killackey E, Jackson H, Gleeson J. Befriending Manual. Melbourne, VIC: Orygen Research Centre, The University of Melbourne; 2003.

20. Sensky T. A randomized controlled trial of cognitive-behavioral therapy for persistent symptoms in schizophrenia resistant to medication. Arch Gen Psychiatry. 2000;57(2):165-172.

21. Kroenke K, Spitzer RL, Williams JB. The PHQ-9: validity of a brief depression severity measure. J Gen Intern Med. 2001;16(9):606-613.

22. Moore M, Ali S, Stuart B, et al. Depression management in primary care: an observational study of management changes related to PHQ-9 score for depression monitoring. Br J Gen Pract. 2012;62(599):e451-e457.

23. Löwe B, Kroenke K, Herzog W, Gräfe K. Measuring depression outcome with a brief self-report instrument: sensitivity to change of the Patient Health Questionnaire (PHQ-9). J Affect Disord. 2004;81(1):61-66.

24. Lowe B, Schenkel I, Carney-Doebbeling C, Gobel C. Responsiveness of the PHQ-9 to psychopharmacological depression treatment. Psychosomatics. 2006;47(1):62-67.

25. Kabacoff RI, Segal DL, Hersen M, Van Hasselt VB. Psychometric properties and diagnostic utility of the Beck Anxiety Inventory and the State-Trait Anxiety Inventory with older adult psychiatric outpatients. J Anxiety Disord. 1997;11(1):33-47.

26. Jones PW. Development and first validation of the COPD assessment test. Eur Respir J. 2009;34(3):648-654.
27. Dodd JW. The COPD assessment test score (CAT) - a multicentre, prospective study of response to pulmonary rehabilitation. Thorax. 2010;65(4 Suppl):S76.

28. Jones PW, Harding G, Wiklund I, et al. Tests of the responsiveness of the COPD assessment test following acute exacerbation and pulmonary rehabilitation. Chest. 2012;142(1):134-140.

29. Larsen DL, Attkission CC, Hargreaves WA, Nguyen TD. Assessment of client/patient satisfaction: development of a general scale. Eval Program Plann. 1979;2:197-207.

30. Horvath AO. Relation between working alliance and outcome in psychotherapy: a meta-analysis. J Couns Psychol. 1991;38(2):139-150.

31. Horwath AO, Greenberg LS. Development and validation of the Working Alliance Inventory. J Counseling Psychology. 1989;36(2):223-233.

32. Tracey TJ, Kokotovic AM. Factor structure of the Working Alliance Inventory. Psychological Assessment; A Journal of Consulting \& Clinical Psychology. 1989;1(3):207-210.

33. Hanson WE. Reliability generalization of working alliance inventory scale scores. Educ Psychol Meas. 2002;62(4):659-673.

34. Charlson ME. A new method of classifying prognostic comorbidity in longitudinal studies: development and validation. J Chronic Dis. 1987; 40(5):373-383.

35. Schulz KF, Altman DG, Moher D. CONSORT 2010 statement: updated guidelines for reporting parallel group randomised trials. Dtsch Med Wochenschr. 2011;136(15):E26.

36. Kessler R. CBT by telephone for depression improved adherence compared with face-to-face CBT in primary care. Ann Intern Med. 2012; 157(6):JC3-12-JC13-12.

37. Bee PE, Lovell K, Lidbetter N, Easton K, Gask L. You can't get anything perfect: "user perspectives on the delivery of cognitive behavioural therapy by telephone." Soc Sci Med. 2010;71(7):1308-1315.
International Journal of COPD

\section{Publish your work in this journal}

The International Journal of COPD is an international, peer-reviewed journal of therapeutics and pharmacology focusing on concise rapid reporting of clinical studies and reviews in COPD. Special focus is given to the pathophysiological processes underlying the disease, intervention programs, patient focused education, and self management protocols.

\section{Dovepress}

This journal is indexed on PubMed Central, MedLine and CAS. The manuscript management system is completely online and includes a very quick and fair peer-review system, which is all easy to use. Visit http://www.dovepress.com/testimonials.php to read real quotes from published authors. 